Published in final edited form as:

Leukemia. 2017 December ; 31(12): 2686-2694. doi:10.1038/leu.2017.152.

\title{
Genetic Deletion of Sost or Pharmacological Inhibition of Sclerostin Prevent Multiple Myeloma-induced Bone Disease without Affecting Tumor Growth
}

\author{
Jesus Delgado-Calle ${ }^{1,5}$, Judith Anderson ${ }^{2}$, Meloney D. Cregor $^{1}$, Keith W. Condon ${ }^{1}$, Stuart

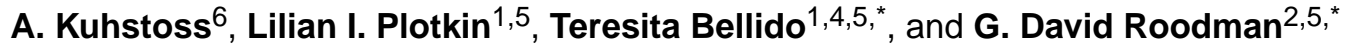 \\ ${ }^{1}$ Department of Anatomy and Cell Biology, Indiana University School of Medicine, Indianapolis, \\ Indiana, USA \\ ${ }^{2}$ Department of Medicine, Division of Hematology/Oncology, Indiana University School of \\ Medicine, Indianapolis, Indiana, USA \\ ${ }^{4}$ Department of Medicine, Division of Endocrinology, Indiana University School of Medicine, \\ Indianapolis, Indiana, USA \\ ${ }^{5}$ Roudebush Veterans Administration Medical Center, Indianapolis, Indiana, USA \\ ${ }^{6}$ Lilly Research Laboratories, Indianapolis, Indiana, USA
}

\section{Abstract}

Multiple myeloma (MM) causes lytic bone lesions due to increased bone resorption and concomitant marked suppression of bone formation. Sclerostin (Scl) levels, an osteocyte-derived inhibitor of $\mathrm{Wnt} / \beta$-catenin signaling, are elevated in MM patient sera and are increased in osteocytes in MM-bearing mice. We show here that genetic deletion of Sost, the gene encoding Scl, prevented MM-induced bone disease in an immune-deficient mouse model of early MM, and that administration of anti-Scl antibody ( $\mathrm{Scl}-\mathrm{Ab}$ ) increased bone mass and decreases osteolysis in immune-competent mice with established MM. Sost/Scl inhibition increased osteoblast numbers, stimulated new bone formation and decreased osteoclast number in MM-colonized bone. Further, Sost/Scl inhibition did not affect tumor growth in vivo or anti-myeloma drug efficacy in vitro. These results identify the osteocyte as a major contributor to the deleterious effects of MM in bone and osteocyte-derived Scl as a promising target for the treatment of established MM-induced bone

Users may view, print, copy, and download text and data-mine the content in such documents, for the purposes of academic research, subject always to the full Conditions of use: http://www.nature.com/authors/editorial_policies/license.html\#terms

"Corresponding authors' information: G. David Roodman, MD, PhD, Department of Medicine, Indiana University School of Medicine, 980 W. Walnut, Suite C312, Indianapolis, Indiana, 46202. Phone: 317-278-6255, Fax: 317-274-0396. groodman@iu.edu. Teresita Bellido, PhD, Department of Anatomy and Cell Biology, and Department of Medicine Indiana University School of Medicine; 635 Barnhill Drive, MS5045A; Indianapolis, IN 46202, USA; Phone: 317-274-7410; Fax: 317-278-2040.

tbellido@iupui.edu.

Competing financial interest

G.D.R. has received consulting honoraria from Amgen Inc. S.A.K is an employee of Lilly Research Laboratories. The remaining authors declare no competing financial interests.

Conflict of interest

G.D.R. has received consulting honoraria from Amgen Inc. S.A.K is an employee of Lilly Research Laboratories. The remaining authors declare no competing financial interests.

Supplementary information is available at Leukemia's website. 
disease. Further, Scl did not interfere with efficacy of chemotherapy for MM suggesting that combined treatment with anti-myeloma drugs and Scl-Ab should effectively control MM growth and bone disease, providing new avenues to effectively control MM and bone disease in patients with active MM.

\section{Introduction}

Multiple myeloma (MM) is characterized by the clonal expansion of malignant plasma cells within the bone marrow, production of monoclonal immunoglobulin (paraprotein), and end organ damage, including lytic lesions in bone, renal dysfunction, hypercalcemia, and anemia $(1,2)$. MM bone disease (MMBD) is characterized by extensive bone resorption and protracted suppression of osteoblast function, resulting in bone lesions that are purely lytic and rarely heal, even in patients with long-term complete remission $(1,2)$. Current antimyeloma regimens have significantly increased the life expectancy for MM patients, but have minor effects on bone repair (3). Therefore, MM patients live longer but continue to suffer from the devastating skeletal sequelae. Thus, new therapeutic regimens that simultaneously target tumor progression and improve MMBD are greatly needed.

MM cell growth, bone disease, and resistance to therapy are highly dependent on the bone/ bone marrow microenvironment. Thus, targeting the interactions between malignant plasma cells and the multiple cell types present in MM microenvironment is required to successfully treat MM $(2,4)$. The contributions of tumor cells, osteoclasts, osteoblasts, stromal cells, and immune cells to MMBD are well known $(1,4,5)$. However, the role of osteocytes in MM is just starting to emerge (6-9). Osteocytes, the most numerous bone cells, are key regulators of physiologic bone remodeling and a major source of factors that regulate osteoclast and osteoblast activity (10-12). Further, dysregulation of osteocyte function and alteration of osteocyte lifespan underlies the pathophysiology of several skeletal disorders (13), and pharmacological targeting of osteocyte-derived factors markedly affects bone homeostasis (14). Recent studies demonstrate that osteocytes are also important contributors to MMBD $(6,9)$.

Sclerostin (Scl), the product of the Sost gene, is a potent Wnt/ $\beta$-catenin antagonist preferentially expressed by osteocytes in the adult skeleton. Scl achieves its bone effects by binding to the lipoprotein receptor-related proteins (LRP) 4/5/6 and blocking Wnt/ $\beta$-catenin signaling to modulate both bone formation and bone resorption (15-17). Neutralizing monoclonal antibodies against $\mathrm{Scl}(\mathrm{Scl}-\mathrm{Ab})$ increase bone mass in both experimental animals and humans, by a dual mechanism that includes increased bone formation and decreased bone resorption $(18,19)$. Thus, Scl has quickly become a promising therapeutic target for the treatment of skeletal diseases characterized by low bone mass.

Since Sost/Scl expression is increased in osteocytes in a murine model of MM (6), and Scl levels are elevated in the sera of MM patients and correlate with reduced osteoblast function and poor patient survival $(20,21)$, we examined the potential of $\mathrm{Scl}$ as a therapeutic target for MMBD. We report that genetic deletion of Sost prevents MM-induced bone loss in an immune-deficient mouse model of early MM, and that treatment with $\mathrm{Scl}-\mathrm{Ab}$ reduces 
osteolysis and increases cancellous bone volume in immune-competent mice with established MM.

\section{Methods}

\section{Antibodies and compounds}

Reagents used in this study are described in Supplementary Methods.

\section{Mouse model of human MM}

Immune-deficient mice with global deletion of Sost ( $\left.\mathrm{Sost}^{-/-} / \mathrm{Scid}\right)$ were generated by crossing Sost ${ }^{-/}$mice (22) with B6.CB17-Prkdcscid/SzJ Scid mice (Jackson laboratories, Bar Harbor, Maine, USA). Six-wk-old Sost ${ }^{-/} / \mathrm{Scid}$ and control littermate mice (wt/Scid) were injected intratibially with $10^{5}$ human JJN3 myeloma cells or saline and sacrificed 4wks later (6). Six to 10 female and male mice per group were used for these experiments. Sixwk-old immune-competent C57BLKaLwRij $(23,24)$ mice were injected intratibially with $10^{5}$ murine 5TGM1 myeloma cells or saline. After four weeks, tumor engraftment was confirmed, mice were stratified by IgG2b levels, and then control mice and mice bearing MM were treated with either Scl-Ab (15mg/kg/wk) or control antibody (IgG) for four additional weeks, when the mice were sacrificed. Seven to 10 female and male mice per group were used for these experiments. Sample size for these studies was calculated based on previous studies $(6,25)$. Mice were fed with a regular diet (Harlan, Indianapolis, IN), received water ad libitum, and were maintained on a 12-hour light/dark cycle. Studies were approved by the Institutional Animal Care and Use Committee of the Indiana University School of Medicine.

\section{Analysis of the skeletal phenotype}

BMD measurements, radiographs, and micro-CT analyses were performed as previously described $(6,26)$. BioQuant software (Nashville, TN) was used to determine osteolytic lesion area. Histomorphometric analyses were performed as previously published (26), and expressed as recommended (27). All these analyses were performed in a blinded fashion.

\section{Serum biochemistry}

Human Kappa Light chain and murine IgG2b circulating levels were determined using enzyme-linked immunosorbent assays (ELISA) kits (Bethyl Laboratories, Inc., Montgomery, TX). N-terminal propeptide of type I procollagen (P1NP) and C-terminal telopeptides of type I collagen (CTX) circulating levels were quantified as previously published (26).

\section{Immunohistochemistry}

Scl, TRAPase and von Kosa staining methods are described in Supplementary Methods.

\section{Cells and culture conditions}

Myeloma cells were treated for 48 hours with BTZ (3-5-10 nM), DEX $\left(10^{-6}-10^{-5}-10^{-4} \mathrm{M}\right)$, or GSI-XX (5-10-20 $\mu \mathrm{M})$ in the presence or absence of Scl-Ab $(5 \mu \mathrm{g} / \mathrm{mL})$. DMSO and IgG 
$(5 \mu \mathrm{g} / \mathrm{mL})$ were used as controls. Viable cells were enumerated by trypan blue exclusion as previously published (6).

\section{Statistics}

Statistical methods are described in Supplementary Methods.

\section{Results \\ Genetic deletion of Sost decreases osteolysis and prevents bone loss induced by MM without affecting MM tumor progression}

We generated mice with a global deletion of Sost in an immune-deficient Scid background (Sost $^{-/-} /$Scid) (Fig. 1a). At 6-wks of age, Sost ${ }^{-/-} /$Scid mice displayed the expected high bone mass phenotype associated with Sost deficiency (22), with increased bone mineral density at all skeletal sites, as well as increased bone volume (BV/TV) and improved bone architecture in the cancellous bone of the tibia (Fig. 1b and 1e). Sost ${ }^{-/} /$Scid and wt/Scid mice injected with human JJN3 myeloma cells had equivalent tumor engraftment (Fig. 1c). However, X-ray analyses revealed that the number and area of osteolytic lesions were reduced in $\mathrm{Sost}^{-/} /$Scid mice by $60 \%$ and 74\%, respectively, compared to wt/Scid mice (Fig. 1d). JJN3-injected wt/Scid mice had a $\sim 50 \%$ decrease in tibia cancellous BV/TV and trabecular number (Tb.N), and a non-significant increase in trabecular separation (Tb.Sp) (Fig. 1e). In contrast, JJN3-injected Sost ${ }^{-/} /$Scid mice displayed no changes in BV/TV or bone architecture compared to saline-injected $\mathrm{Sost}^{-/} /$Scid mice. These results demonstrate that Sost deficiency in vivo protects mice from MM-induced bone loss without affecting tumor growth.

\section{Sost $^{-/-/ S c i d ~ m i c e ~ a r e ~ p r o t e c t e d ~ f r o m ~ t h e ~ d e c r e a s e ~ i n ~ o s t e o b l a s t ~ n u m b e r ~ a n d ~ f u n c t i o n ~}$ induced by MM}

To establish the cellular mechanisms underlying the protective effects of Sost deficiency on bone mass in mice bearing MM, osteoblasts and osteoclasts numbers, and osteoblast function were quantified in the cancellous bone of the proximal tibia (Fig 2a). Sost ${ }^{-/} /$Scid mice exhibited increased bone formation (BFR/BS) and osteoblasts (Fig. 2a and 2b), but no changes in osteoclasts (Fig. 2c), compared to control wt/Scid littermates. JJN3-injected wt/ Scid mice displayed decreased mineral apposition (MAR) and BFR (Fig. 2a), reduced bone surface covered by osteoblasts (Ob.S/BS) and lower numbers of osteoblasts (Ob.N/BS) (Fig. 2b), as well as increased osteoclast surface (Oc.S/BS) and number (Oc.N/BS) (Fig. 2c). Strikingly, osteoblast surface/number or osteoblast function remained elevated in JJN3injected Sost ${ }^{-/} /$Scid mice and indistinguishable from saline-injected Sost ${ }^{-/} /$Scid mice (Fig. 2b). In addition, JJN3-injected Sost ${ }^{-/} /$Scid mice had increased osteoclasts compared to saline-injected $\mathrm{Sost}^{-/} /$Scid mice, although the results did not reach statistical significance (Fig. 2c). These results demonstrate that $\mathrm{Scl}$ contributes to the decrease in osteoblast number and function and the increase in osteoclasts induced by myeloma cells. 


\section{Administration of Scl-Ab reduces osteolysis and increases cancellous bone mass in mice with established MM, without altering tumor growth}

We next examined the effect of pharmacological inhibition of Scl in an immune-competent mouse model of established MM (Fig. 3a). C57BL/KaLwRij mice injected with murine 5TGM1 myeloma cells exhibited 2 -fold increase in the levels of IgG2b 4-wks after myeloma cell injection $(0.36 \pm 0.01$ vs. $0.08 \pm 0.02$, saline- and 5TGM1-injected respectively, $\mathrm{p} \leq 0.01$ ). After 4-wks of treatment, serum IgG2b levels were similar in 5TGM1-injected mice receiving Scl-Ab or IgG (Fig. 3b). No evidence of extramedullary disease was detected and the distribution of 5TGM1 myeloma cells in the marrow cavity of mice receiving Scl-Ab and $\mathrm{IgG}$ was similar (Fig. 3c). 5TGM1-injected mice had increased numbers of Scl-positive osteocytes in cortical and cancellous bone (Fig. 3d), that was not altered by Scl-Ab administration.

Scl-Ab reduced the number of osteolytic lesions by $46 \%$ compared to the IgG-treated mice (Fig. 3e). Further, 5TGM1-injected mice and receiving IgG injections had $\sim 35 \%$ decreased $\mathrm{BV} / \mathrm{TV}$ and Tb.N, and increased Tb.Sp (Fig. 3f). In contrast, mice receiving Scl-Ab, regardless of whether they were injected with 5TGM1 myeloma cells or not, displayed increased trabecular BV/TV (52\%), Tb.N (22\%), Tb.Th (33\%) and decreased Tb.Sp (14\%) compared to saline-injected mice receiving $\operatorname{IgG}$ (Fig. 3f). Taken together, these results support that $\mathrm{Scl}$ contributes to bone loss in mice with established MM, but not to MM growth.

\section{Scl-Ab therapy reduces osteolysis and increases cancellous bone mass in mice with established MM}

We next investigated the cellular basis for the bone gain in mice bearing MM treated with Scl-Ab. In saline-injected mice, Scl-Ab increased osteoblast surface and number and the serum levels of the bone formation marker P1NP (Fig. 4a and c); and decreased osteoclast surface, without significant changes in osteoclast numbers or in the circulating levels of the bone resorption marker CTX (Fig. 4b and c). Mice with established MM and receiving IgG exhibited decreased osteoblast surface/number and a non-significant decrease in serum P1NP (Fig. 4a), and increased osteoclast surface/number but no detectable changes in serum CTX (Fig. 4b). Scl-Ab similarly increased osteoblast surface/number and serum P1NP in 5TGM1-injected mice or saline-injected mice (Fig. 4a). Further, 5TGM1-injected mice receiving Scl-Ab still exhibited increased osteoclast surface and osteoclast number, although these changes did not reach statistical significance (Fig. 4b). Thus, Scl-Ab treatment increases osteoblast number and function and partially decreases osteoclasts in bones colonized by myeloma cells.

\section{Scl-Ab does not alter the anti-MM activity of Bortezomib, Dexamethasone, or a Notch inhibitor}

As expected, BTZ, DEX and GSIXX decreased the number of viable JJN3 and 5TGM1 myeloma cells in a dose-dependent manner (Fig. 5a). Consistent with the lack of effect of the Scl-Ab on MM growth in vivo, Scl-Ab did not affect the viability of JJN3 (Fig. 5b) or 5TGM1 (Fig. 5c) myeloma cells. Further, the inhibitory effect on myeloma cell viability induced by BTZ, DEX, or GSIXX remained intact when combined with Scl-Ab. These 
results demonstrate that Scl-Ab does not affect myeloma cell viability and does not interfere with the efficacy of anti-MM drugs to induce myeloma cell death in vitro.

\section{Discussion}

Recent advances in the treatment of MM have significantly increased patient survival; however, the skeletal sequelae persist even in patients with complete remission (2). Bisphosphonates, the mainstay of MMBD therapy, prevent bone loss but further decrease bone formation and adversely affect bone quality (28). Therefore, agents that build new bone and improve MMBD but do not increase MM growth are an unmet need. Here we show that in bone colonized by myeloma cells, osteocytes overproduce $\mathrm{Scl}$, an antagonist of $\mathrm{Wnt} / \beta$ catenin signaling that potently inhibits bone formation (Fig. 6) (17). Genetic deletion of Sost in immune-deficient mice prevents MMBD in the early phase of MM, and pharmacological inhibition of Scl increases bone formation and decreases osteolysis in immune-competent mice with established active MM. Mechanistic studies demonstrated that Sost/Scl inhibition overcame MM-induced osteoblast suppression, stimulated new bone formation, and partially blocked the increase in osteoclasts. Further, inhibition of Sost/Scl did not alter tumor growth in vivo or the efficacy of anti-myeloma drugs in vitro. Taken together, these results highlight the importance of Scl production in the MM microenvironment and provide the rationale for combining anti-myeloma drugs with Scl-Ab to simultaneously prevent tumor progression and stimulate osteoblast function to improve MMBD.

Our findings support the notion that overproduction of Scl by osteocytes participates in the suppression of new bone formation in MM. Multiple causes contribute to osteoblast inhibition in MM, including several Wnt inhibitors, blockade of Runx2, and secreted factors $(29,30)$. Activation of Wnt signaling induces osteogenesis by directly stimulating Runx 2 in osteoblasts and enhances osteoblast/osteocyte survival $(31,32)$. Thus, it is likely that Scl-Ab overcomes MM-induced Runx2 blockade as well as prevents osteoblast/osteocyte apoptosis. Moreover, Scl-Ab therapy also increases bone formation on quiescent surfaces via modeling-based bone formation that does not require previous osteoclast activity $(18,33$, 34). Therefore, it is possible that the effects observed with Scl-Ab therapy in our model of MM are due to recruitment of unaffected osteoblasts or activation of bone lining cells covering quiescent bone surfaces to become bone forming cells (35). In addition, we recently demonstrated that activation of $\beta$-catenin signaling in osteocytes is sufficient to stimulate osteoblast differentiation and matrix synthesis (11), suggesting that autocrine effects of Wnt signaling in osteocytes could also contribute. Further studies are warranted to identify the specific mechanisms by which Scl-Ab overcomes osteoblast suppression in MM. Moreover, whether the Scl-Ab prevented the development of new lesions or promoted the filling of eroded areas was not addressed in our study and warrants future investigation.

Consistent with the current study, an earlier report showed that Scl-Ab prevented the decrease in bone volume in a mouse model of early MM (21). However, the cellular mechanism(s) underlying this effect was not studied. Herein, we demonstrate that the increases in bone mass induced by Sost/Scl inhibition result mainly from increased osteoblasts and stimulation of their function, resulting in new bone formation even in areas colonized by myeloma cells. Indeed, the protective bone effects are independent of the 
presence of MM cells, as mice with low and high tumor burden were equally protected (Suppl. Fig. 1).

Our results also suggest that the increased Scl levels in the MM microenvironment may have a direct role inhibiting osteoclast differentiation, thus potentially contributing to the improvement of MMBD. However, no changes in circulating levels of the bone resorption marker CTX were detected, likely due to restriction of the tumor cells to the marrow compartment of the injected tibia. Nevertheless, these results are consistent with previous findings from our laboratory showing that $\mathrm{Sost}^{-/-} / \mathrm{Scid}$ mice are protected from GC-induced bone resorption (36), and with clinical and animal data demonstrating that the bone gain achieved by Scl-Ab therapy, although primarily due to stimulation of osteoblast activity, is accompanied by inhibition of bone resorption $(37,38)$. Multiple mechanism(s) may account for the inhibitory effects of Scl-Ab on osteoclasts. Inhibition of Sost/Scl, with the consequent increased $\mathrm{Wnt} / \beta$-catenin signaling, could modulate resorption by upregulating OPG $(15,39)$. Scl also upregulates the expression of receptor activator of nuclear factor kappa-B ligand (RANKL) and increases osteoclast formation in vitro and in vivo $(11,40)$. Thus, Scl-Ab may reduce RANKL expression and, through inhibition of Wnt/ $\beta$-catenin signaling, also directly inhibit osteoclast precursor differentiation (41).

Studies by Yaccoby and co-workers suggested that myeloma cells suppress osteoblast differentiation thereby blocking the anti-myeloma effects of mature osteoblasts, and that bone anabolic therapies not only improve MMBD, but also simultaneously control MM progression (42). However, we found that increased osteoblast numbers and function did not change MM growth in vivo or MM cell viability in vitro. Thus, the effect of osteoblasts on MM progression remains as an open question. Our findings that the increased osteoblast number induced by Sost/Scl inhibition does not interfere with tumor engraftment/growth are consistent with a recent report showing no differences in myeloma burden in mice with early stage MM treated with Scl-Ab and the lack of additive effects on MM growth when Scl-Ab was combined with Bortezomib (21). Similarly, neutralization of Dickkopf-related protein (DKK-1) in a murine model of MM abolished the suppression of osteoblastogenesis by MM, but had variable effects on tumor burden $(43,44)$. The stimulatory effects of Bortezomib on bone cells may contribute to the anti-myeloma properties of this drug (45). Thus, it is possible that different mechanisms underlie the actions of distinct bone anabolic therapies, and therefore some of them could exhibit anti-myeloma effects. Because Scl-Ab decreased MM-induced bone resorption, we cannot exclude the possibility that prolonged therapy with Scl-Ab may have anti-myeloma effects by affecting osteoclast support of MM growth, or increase the susceptibility of myeloma cells to anti-myeloma therapy when combined with other anti-myeloma agents.

Our data showing that $\mathrm{Scl}-\mathrm{Ab}$ does not interfere with drugs that induce MM cell apoptosis via diverse mechanisms suggest that therapies combining Scl-Ab with anti-myeloma drugs could result in both beneficial skeletal outcomes and inhibition of tumor progression.

Additionally, Scl-Ab therapy may prevent some of the adverse skeletal effects of antimyeloma drugs, such as glucocorticoids (46-48), as genetic deletion of Sost protects from glucocorticoid-induced bone loss (36). 
In conclusion, our study demonstrates that $\mathrm{Scl}$ is a promising target for the treatment of MMBD. Scl-Ab therapy stimulates new bone formation in areas colonized by myeloma cells, thus providing potential new avenues to improve bone disease in patients with active MM. Whether patients in complete remission or patients with monoclonal gammopathy of undetermined significance could benefit from pharmacological inhibition of Scl remains to be determined. Further, the current results support the notion that osteocytes contribute to the generation of a microenvironment that is favorable to MM progression and bone destruction, and suggest that targeting osteocytes and their derived factors represents a new and promising approach for the treatment of MM.

\section{Supplementary Material}

Refer to Web version on PubMed Central for supplementary material.

\section{Acknowledgments}

We thank Kevin McAndrews, Amy Y. Sato, and Dan Zhou for assistance in tissue collection. Scl-Ab was provided by Eli Lilly (Indianapolis, IN). Sost ${ }^{-1-}$ mice were provided by Amgen Inc. (Thousand Oaks, CA) and UCB (Brussels, Belgium). This work was supported by the NIH (Indiana-CTSI P30, 1R21CA179017-02 and R01AR059679 to GDR; R01AR059357, R01 DK076007, and S10-RR023710 to TB, and R01 CA209882 to GDR and TB), the VA (Merit Review to TB and to GDR). JDC received an IBMS Gideon and Sevgi Rodan Fellowship, a Scholar Award from ASH, and a Brian D. Novis Junior Research Grant Award from IMFR.

\section{Reference List}

1. Roodman GD. Pathogenesis of myeloma bone disease. J Cell Biochem. 2010; 109:283-291. [PubMed: 20014067]

2. Bianchi G, Munshi NC. Pathogenesis beyond the cancer clone(s) in multiple myeloma. Blood. 2015; 125:3049-3058. [PubMed: 25838343]

3. Silbermann R, Roodman GD. Bone effects of cancer therapies: pros and cons. Curr Opin Support Palliat Care. 2011; 5:251-257. [PubMed: 21768880]

4. Roodman GD. Targeting the bone microenvironment in multiple myeloma. J Bone Miner Metab. 2010; 28:244-250. [PubMed: 20127498]

5. Roodman GD. Role of the bone marrow microenvironment in multiple myeloma. J Bone Miner Res. 2002; 17:1921-1925. [PubMed: 12412796]

6. Delgado-Calle J, Anderson J, Cregor MD, Hiasa M, Chirgwin JM, Carlesso N, et al. Bidirectional Notch signaling and osteocyte-derived factors in the bone marrow microenvironment promote tumor cell proliferation and bone destruction in multiple myeloma. Cancer Res. 2016; 76:1089-1100. [PubMed: 26833121]

7. Delgado-Calle J, Bellido T, Roodman GD. Role of osteocytes in multiple myeloma bone disease. Curr Opin Support Palliat Care. 2014; 8:407-413. [PubMed: 25289928]

8. Toscani D, Palumbo C, Dalla PB, Ferretti M, Bolzoni M, Marchica V, et al. The Proteasome Inhibitor Bortezomib Maintains Osteocyte Viability in Multiple Myeloma Patients by Reducing Both Apoptosis and Autophagy: A New Function for Proteasome Inhibitors. J Bone Miner Res. 2016; 31:815-827. [PubMed: 26551485]

9. Giuliani N, Ferretti M, Bolzoni M, Storti P, Lazzaretti M, Dalla PB, et al. Increased osteocyte death in multiple myeloma patients: role in myeloma-induced osteoclast formation. Leukemia. 2012; 26:1391-1401. [PubMed: 22289923]

10. Dallas SL, Prideaux M, Bonewald LF. The osteocyte: an endocrine cell ... and more. Endocr Rev. 2013; 34:658-690. [PubMed: 23612223]

11. Tu X, Delgado-Calle J, Condon KW, Maycas M, Zhang H, Carlesso N, et al. Osteocytes mediate the anabolic actions of canonical Wnt/ $\beta$-catenin signaling in bone. Proc Natl Acad Sci U S A. 2015; 112:E478-E486. [PubMed: 25605937] 
12. Bellido T. Osteocyte-Driven Bone Remodeling. Calcif Tissue Int. 2013; 94:25-34. [PubMed: 24002178]

13. Delgado-Calle J, Bellido T. Osteocytes and Skeletal Pathophysiology. Curr Mol Biol Rep. 2015; 1:157-167. [PubMed: 26693137]

14. Plotkin LI, Bellido T. Osteocytic signalling pathways as therapeutic targets for bone fragility. Nat Rev Endocrinol. 2016; 12:593-605. [PubMed: 27230951]

15. Baron R, Kneissel M. WNT signaling in bone homeostasis and disease: from human mutations to treatments. Nat Med. 2013; 19:179-192. [PubMed: 23389618]

16. Poole KE, Van Bezooijen RL, Loveridge N, Hamersma H, Papapoulos SE, Lowik CW, et al. Sclerostin is a delayed secreted product of osteocytes that inhibits bone formation. FASEB J. 2005; 19:1842-1844. [PubMed: 16123173]

17. Delgado-Calle J, Sato AY, Bellido T. Role and mechanism of action of sclerostin in bone. Bone. 2017; 96:29-37. [PubMed: 27742498]

18. Ominsky MS, Boyce RW, Li X, Ke HZ. Effects of sclerostin antibodies in animal models of osteoporosis. Bone. 2017; 96:63-75. [PubMed: 27789417]

19. Ke HZ, Richards WG, Li X, Ominsky MS. Sclerostin and Dickkopf-1 as Therapeutic Targets in Bone Diseases. Endocr Rev. 2012; 33:747-783. [PubMed: 22723594]

20. Terpos E, Christoulas D, Katodritou E, Bratengeier C, Gkotzamanidou M, Michalis E, et al. Elevated circulating sclerostin correlates with advanced disease features and abnormal bone remodeling in symptomatic myeloma: reduction post-bortezomib monotherapy. Int J Cancer. 2012; 131:1466-1471. [PubMed: 22052418]

21. Eda H, Santo L, Wein MN, Hu DZ, Cirstea DD, Nemani N, et al. Regulation of Sclerostin Expression in Multiple Myeloma by Dkk-1; A Potential Therapeutic Strategy for Myeloma Bone Disease. J Bone Miner Res. 2016; 31:1225-1234. [PubMed: 26763740]

22. Li X, Ominsky MS, Niu QT, Sun N, Daugherty B, D’Agostin D, et al. Targeted deletion of the sclerostin gene in mice results in increased bone formation and bone strength. J Bone Miner Res. 2008; 23:860-869. [PubMed: 18269310]

23. Asosingh K, Radl J, Van RI, Van CB, Vanderkerken K. The 5TMM series: a useful in vivo mouse model of human multiple myeloma. Hematol J. 2000; 1:351-356. [PubMed: 11920212]

24. Radl J, Croese JW, Zurcher C, Van den Enden-Vieveen MH, de Leeuw AM. Animal model of human disease. Multiple myeloma Am J Pathol. 1988; 132:593-597. [PubMed: 3414786]

25. D'Souza S, del PD, Jin S, Sun Q, Huston AJ, Kostov FE, et al. Gfi1 expressed in bone marrow stromal cells is a novel osteoblast suppressor in patients with multiple myeloma bone disease. Blood. 2011; 118:6871-6880. [PubMed: 22042697]

26. Delgado-Calle J, Tu X, Pacheco-Costa R, McAndrews K, Edwards R, Pellegrini G, et al. Control of bone anabolism in response to mechanical loading and PTH by distinct mechanisms downstream of the PTH receptor. J Bone Miner Res. 2017; 32:522-535. [PubMed: 27704638]

27. Dempster DW, Compston JE, Drezner MK, Glorieux FH, Kanis JA, Malluche H, et al. Standardized nomenclature, symbols, and units for bone histomorphometry: A 2012 update of the report of the ASBMR Histomorphometry Nomenclature Committee. J Bone Miner Res. 2013; 28:2-17. [PubMed: 23197339]

28. Papamerkouriou YM, Kenanidis E, Gamie Z, Papavasiliou K, Kostakos T, Potoupnis M, et al. Treatment of multiple myeloma bone disease: experimental and clinical data. Expert Opin Biol Ther. 2015; 15:213-230. [PubMed: 25388648]

29. Silbermann R, Roodman GD. Myeloma bone disease: Pathophysiology and management. J Bone Oncol. 2013; 2:59-69. [PubMed: 26909272]

30. Giuliani N, Colla S, Morandi F, Lazzaretti M, Sala R, Bonomini S, et al. Myeloma cells block RUNX2/CBFA1 activity in human bone marrow osteoblast progenitors and inhibit osteoblast formation and differentiation. Blood. 2005; 106:2472-2483. [PubMed: 15933061]

31. Gaur T, Lengner CJ, Hovhannisyan H, Bhat RA, Bodine PV, Komm BS, et al. Canonical WNT signaling promotes osteogenesis by directly stimulating Runx2 gene expression. J Biol Chem. 2005; 280:33132-33140. [PubMed: 16043491]

32. Bodine PV. Wnt signaling control of bone cell apoptosis. Cell Res. 2008; 18:248-253. [PubMed: 18212734] 
33. Li X, Niu QT, Warmington KS, Asuncion FJ, Dwyer D, Grisanti M, et al. Progressive increases in bone mass and bone strength in an ovariectomized rat model of osteoporosis after 26 weeks of treatment with a sclerostin antibody. Endocrinology. 2014; 155:4785-4797. [PubMed: 25259718]

34. Li X, Ominsky MS, Warmington KS, Niu QT, Asuncion FJ, Barrero M, et al. Increased Bone Formation and Bone Mass Induced by Sclerostin Antibody Is Not Affected by Pretreatment or Cotreatment with Alendronate in Osteopenic, Ovariectomized Rats. Endocrinology. 2011; 152:3312-3322. [PubMed: 21733832]

35. Kim SW, Lu Y, Williams EA, Lai F, Lee JY, Enishi T, et al. Sclerostin Antibody Administration Converts Bone Lining Cells into Active Osteoblasts. J Bone Miner Res. 2016; e-pub ahead of print 14 November 2016. doi: 10.1002/jbmr.3038

36. Sato AY, Cregor M, Delgado-Calle J, Condon KW, Allen MR, Peacock M, et al. Protection from glucocorticoid-induced osteoporosis by anti-catabolic signaling in the absence of Sost/sclerostin. J Bone Miner Res. 2016; 31:1791-1802. [PubMed: 27163932]

37. Padhi D, Allison M, Kivitz AJ, Gutierrez MJ, Stouch B, Wang C, et al. Multiple doses of sclerostin antibody romosozumab in healthy men and postmenopausal women with low bone mass: a randomized, double-blind, placebo-controlled study. J Clin Pharmacol. 2014; 54:168-178. [PubMed: 24272917]

38. McClung MR, Grauer A, Boonen S, Bolognese MA, Brown JP, ez-Perez A, et al. Romosozumab in Postmenopausal Women with Low Bone Mineral Density. N Engl J Med. 2014; 370:412-420. [PubMed: 24382002]

39. Glass DA, Bialek P, Ahn JD, Starbuck M, Patel MS, Clevers H, et al. Canonical Wnt signaling in differentiated osteoblasts controls osteoclast differentiation. Dev Cell. 2005; 8:751-764. [PubMed: 15866165]

40. Wijenayaka AR, Kogawa M, Lim HP, Bonewald LF, Findlay DM, Atkins GJ. Sclerostin stimulates osteocyte support of osteoclast activity by a RANKL-dependent pathway. PLoS ONE. 2011; 6:e25900. [PubMed: 21991382]

41. Wei W, Zeve D, Suh JM, Wang X, Du Y, Zerwekh JE, et al. Biphasic and dosage-dependent regulation of osteoclastogenesis by beta-catenin. Mol Cell Biol. 2011; 31:4706-4719. [PubMed: 21876000]

42. Yaccoby S. Osteoblastogenesis and tumor growth in myeloma. Leuk Lymphoma. 2010; 51:213220. [PubMed: 20038269]

43. Yaccoby S, Ling W, Zhan F, Walker R, Barlogie B, Shaughnessy JD Jr. Antibody-based inhibition of DKK1 suppresses tumor-induced bone resorption and multiple myeloma growth in vivo. Blood. 2007; 109:2106-2111. [PubMed: 17068150]

44. Heath DJ, Chantry AD, Buckle CH, Coulton L, Shaughnessy JD Jr, Evans HR, et al. Inhibiting Dickkopf-1 (Dkk1) removes suppression of bone formation and prevents the development of osteolytic bone disease in multiple myeloma. J Bone Miner Res. 2009; 24:425-436. [PubMed: 19016584]

45. Qiang YW, Hu B, Chen Y, Zhong Y, Shi B, Barlogie B, et al. Bortezomib induces osteoblast differentiation via Wnt-independent activation of beta-catenin/TCF signaling. Blood. 2009; 113:4319-4330. [PubMed: 19196662]

46. Terpos E, Berenson J, Raje N, Roodman GD. Management of bone disease in multiple myeloma. Expert Rev Hematol. 2014; 7:113-125. [PubMed: 24433088]

47. Eshaghian S, Berenson JR. Multiple myeloma: improved outcomes with new therapeutic approaches. Curr Opin Support Palliat Care. 2012; 6:330-336. [PubMed: 22871979]

48. Weinstein RS. Clinical practice. Glucocorticoid-induced bone disease. N Engl J Med. 2011; 365:62-70. [PubMed: 21732837] 

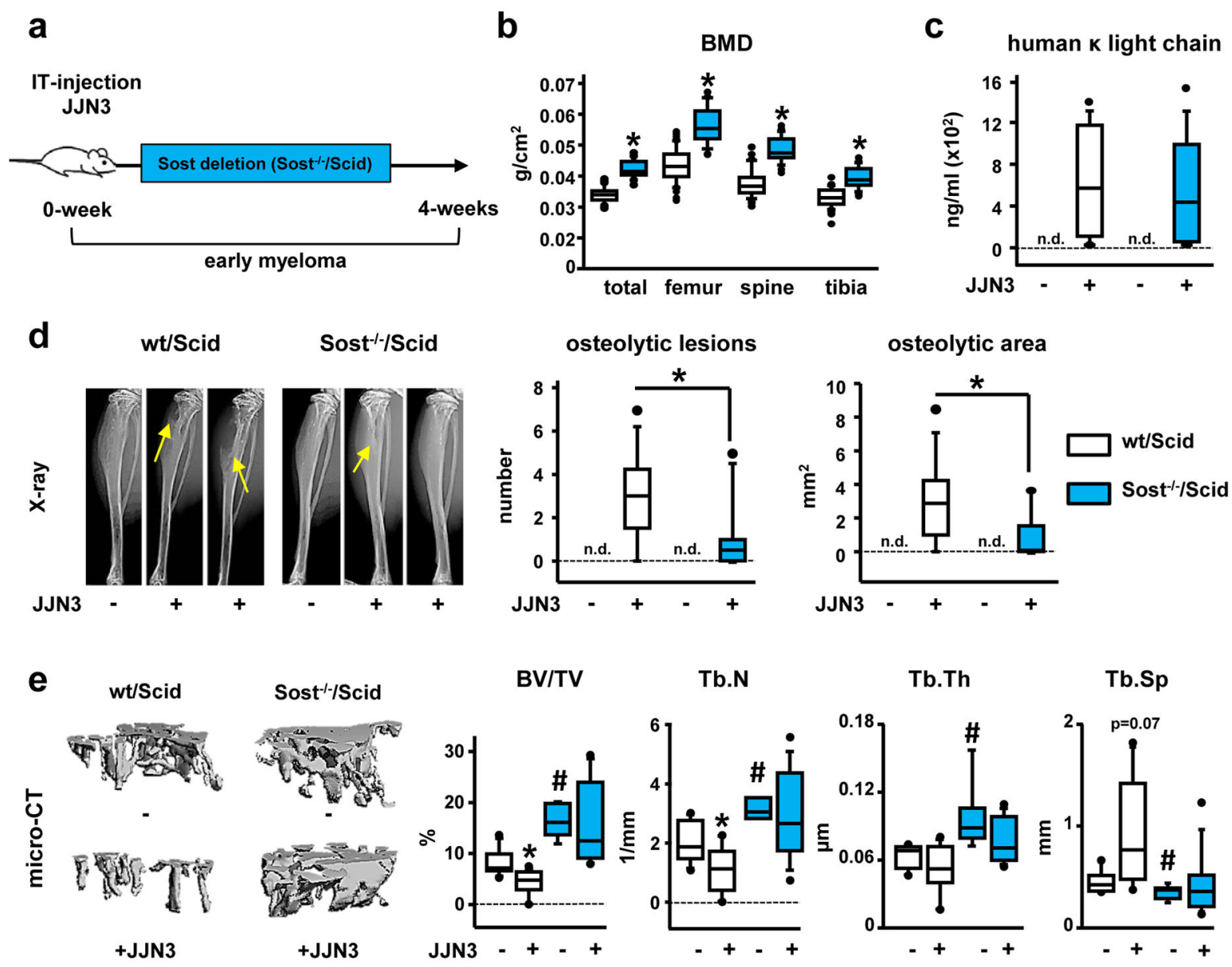

Tb.Th

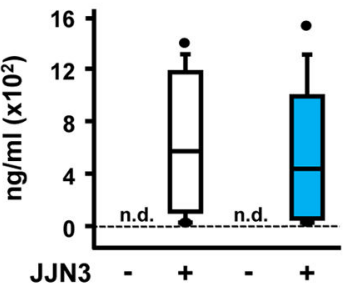

Figure 1. Genetic deletion of Sost decreases osteolysis and prevents bone loss induced by MM tumors without affecting tumor growth

(a) Experimental design (IT-intratibial injection). (b) Bone mineral density (BMD); *p $₫ 0.05$ vs wt/Scid mice. (c) Serum human Kappa light chain 4-wks after cell inoculation (n.d., not detected). (d) Tibia X-rays and number/area of osteolytic lesions (n.d., not detected) $* \mathrm{p} \unlhd 0.05 \mathrm{vs}$ wt/Scid JJN3-injected mice. (e) MicroCT images and microarchitecture of proximal tibia cancellous bone; \# p $₫ 0.05$ vs wt/Scid (saline); *p $₫ 0.05$ vs saline-injected mice. Saline/JJN3-injected: $n=7 / 9 \mathrm{wt} / \mathrm{Scid}$ and $n=6 / 10 \mathrm{Sost}^{-/}-/$Scid mice. Box plots: middle line in box represents the median, whiskers the $95 \%$ confidence interval of the mean, and circles are outliers from the $95 \%$ confidence interval. (BV/TV) is bone volume over tissue volume; (Tb.N) is trabecular number; (Tb.Th) is trabecular thickness and (Tb.Sp) is trabecular separation. 
a

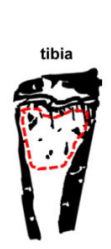

b

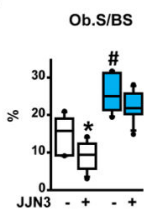

Ob.S/BS

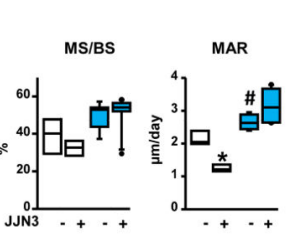

Ob.N/BS

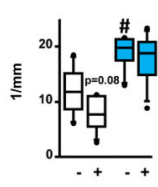

Von Kossa staining
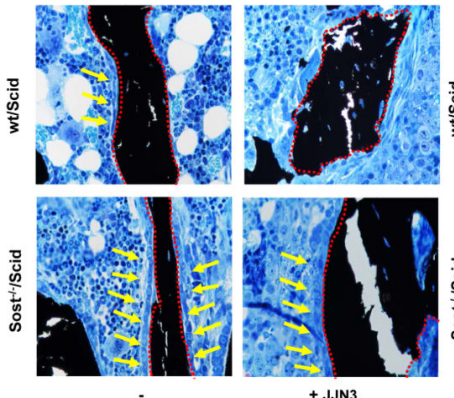

- JJN3

$\square$ wt/Scid $\square$ Sost't/Scid

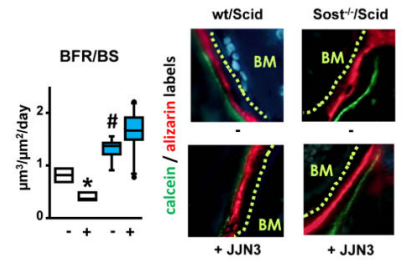

c

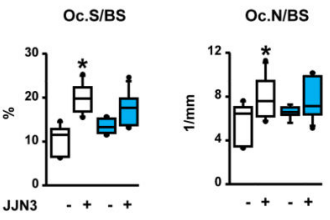

TRAP staining
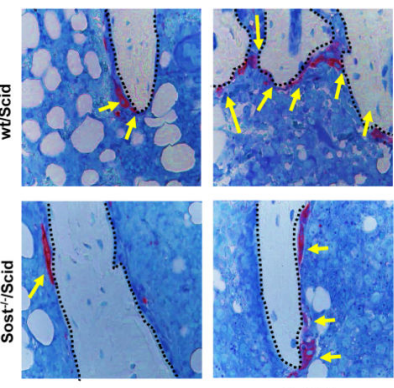

+ JJN3

Figure 2. Genetic deletion of Sost prevents the decrease in bone formation induced by myeloma cells

(a) Region of interest analyzed: cancellous bone of the proximal tibia and dynamic histomorphometric indexes and representative images of labeled bone surfaces; saline/JJN3injected: $n=4 / 4 \mathrm{wt} / \mathrm{Scid}$ and $\mathrm{n}=5 / 6 \mathrm{Sost}^{-/} /$Scid mice; \# $\mathrm{p} \unlhd 0.05 \mathrm{vs} \mathrm{wt} / \mathrm{Scid}$ (saline); *p $₫ 0.05$ vs saline injected mice. Static hystomorphometric quantification of osteoblasts (b) and osteoclasts (c) on bone stained with von Kossa and TRAPase; saline/JJN3-injected: $n=7 / 9$ $\mathrm{wt} / \mathrm{Scid}$ and $\mathrm{n}=6 / 10 \mathrm{Sost}^{-/}-/$Scid mice; ${ }^{*} \mathrm{p} \unlhd 0.05$ vs saline-injected mice. In figure $\mathbf{b}$, red dotted lines indicate bone surfaces and yellow arrows point at osteoblasts. In figure c, black dotted lines indicate bone surfaces and yellow arrows point at TRAP positive osteoclasts. Abbreviations are as follows: Mineralizing surface over bone surface (MS/BS); mineral apposition rate (MAR); bone formation rate over bone surface (BFR/BS); osteoblast surface over bone surface (Ob.S/BS); osteoblast number over bone surface (Ob.N/BS); osteoclast surface over bone surface (Oc.S/BS); osteoblast number over bone surface (Oc.N/BS). 

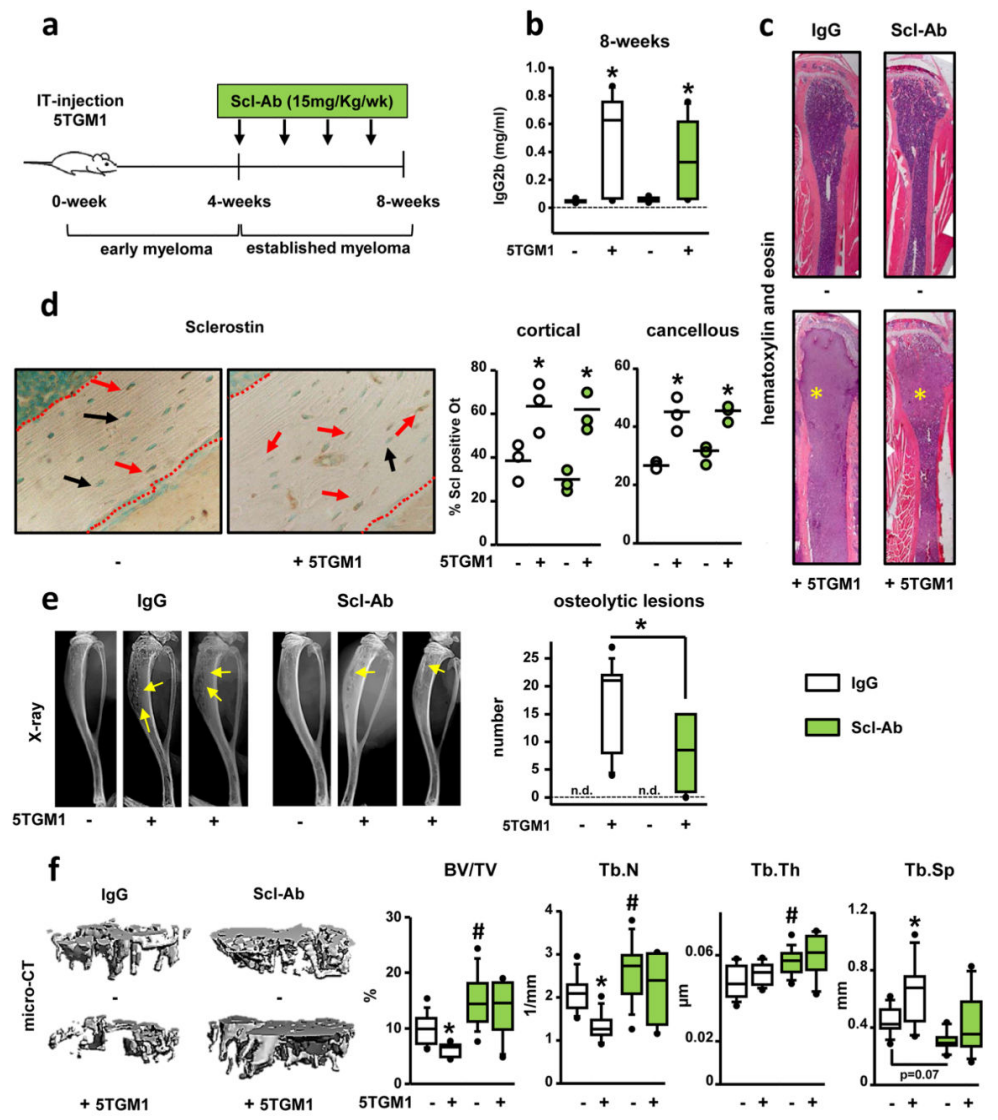

Figure 3. Treatment with Scl-Ab reduces the number of osteolytic lesions and increases cancellous bone mass in mice with established MM disease

(a) Experimental design. (b) Serum IgG2b at 8-wks; saline/5TGM1-injected: $\mathrm{n}=9 / 9 \mathrm{IgG}$ and $\mathrm{n}=10 / 7 \mathrm{Scl}-\mathrm{Ab}$; *p $₫ 0.05$ vs saline injected mice. (c) Hematoxylin/eosin staining of the tibia; asterisk indicates the MM tumors. (d) Images and quantification of Scl positive osteocytes ( $\mathrm{Scl}+\mathrm{ve} \mathrm{Ot}$ ) in cortical and cancellous bone; saline/5TGM1-injected: $\mathrm{n}=3 / 3 \mathrm{IgG}$ and $n=3 / 3$ $\mathrm{Scl}-\mathrm{Ab} ;{ }^{*} \mathrm{p} \triangle 0.05$ vs saline injected mice; red dotted lines indicate bone surfaces, red arrows point to Scl+ve Ot, and black arrows point to Scl-ve Ot. (e) Tibia X-rays and number of osteolytic lesions at 8-wks (n.d., not detected); saline/5TGM1-injected: $\mathrm{n}=9 / 9 \mathrm{IgG}$ and $\mathrm{n}=10 / 7 \mathrm{Scl}-\mathrm{Ab}$; *p $₫ 0.05$ vs 5TGM1-injected mice receiving IgG. (f) MicroCT images and bone microarchitecture; saline/5TGM1-injected: $\mathrm{n}=9 / 9 \mathrm{IgG}$ and n=10/7 Scl-Ab; \# p $₫ .05$ vs IgG-treated (saline) mice; *p $₫ 0.05$ vs saline-injected mice. 

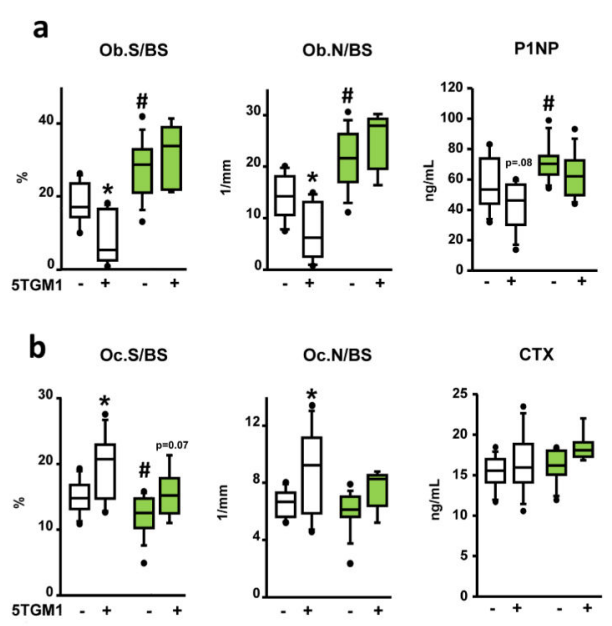

c

TRAP staining

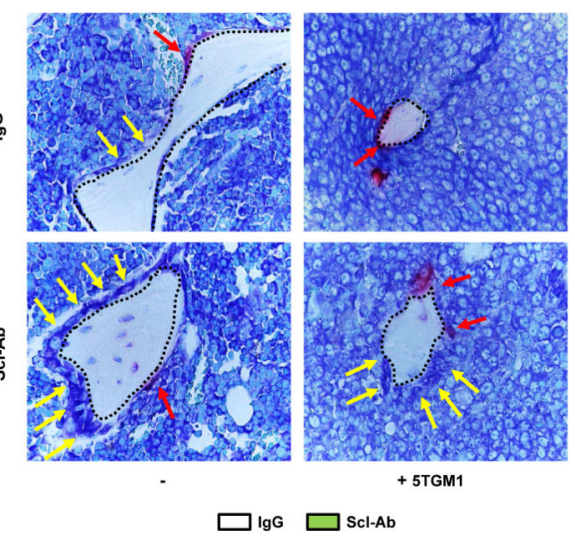

Figure 4. Scl-Ab treatment increases osteoblasts in mice with established MM disease Static histomorphometry quantification of osteoblasts (a) and osteoclasts (b) in bone of mice treated with IgG or Scl-Ab; \# p $₫ 0.05$ vs IgG-treated (saline) mice; *p $₫ 0.05$ vs salineinjected mice. Serum levels of P1NP (a) and CTX (b); \# p $₫$ ).05 vs IgG-treated (saline) mice; *p $₫ 0.05$ vs saline-injected mice. Saline/5TGM1-injected: $n=9 / 9$ IgG and n=10/7 Scl$\mathrm{Ab}(\mathbf{c})$ Black dotted lines indicated bone surfaces, yellow arrows point at osteoblasts, and red arrows point at TRAP positive osteoclasts. 

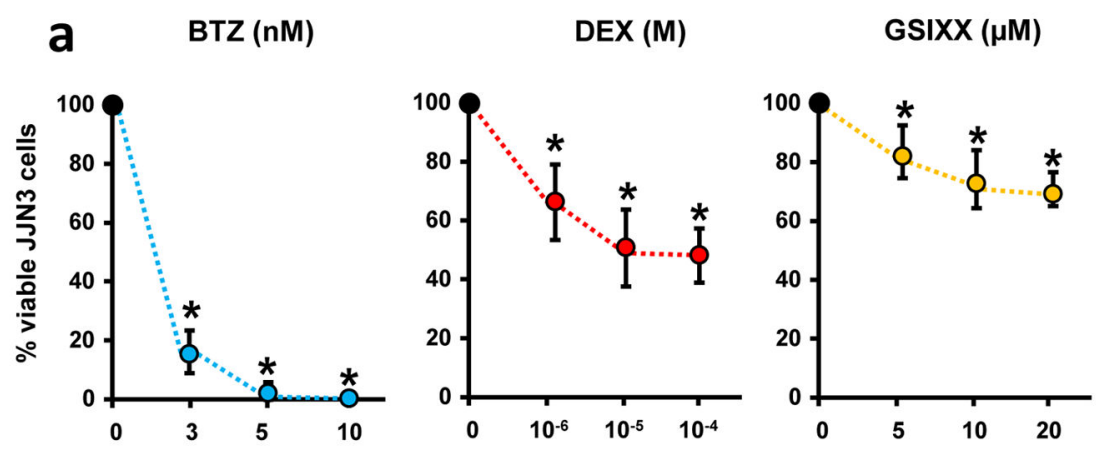

b

viable JJN3 cells

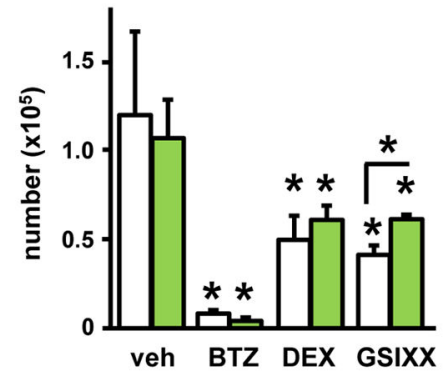

$\mathrm{BTZ}(\mathrm{nM})$

DEX (M)

$\operatorname{GSIXX}(\mu \mathrm{M})$

C

viable 5TGM1 cells
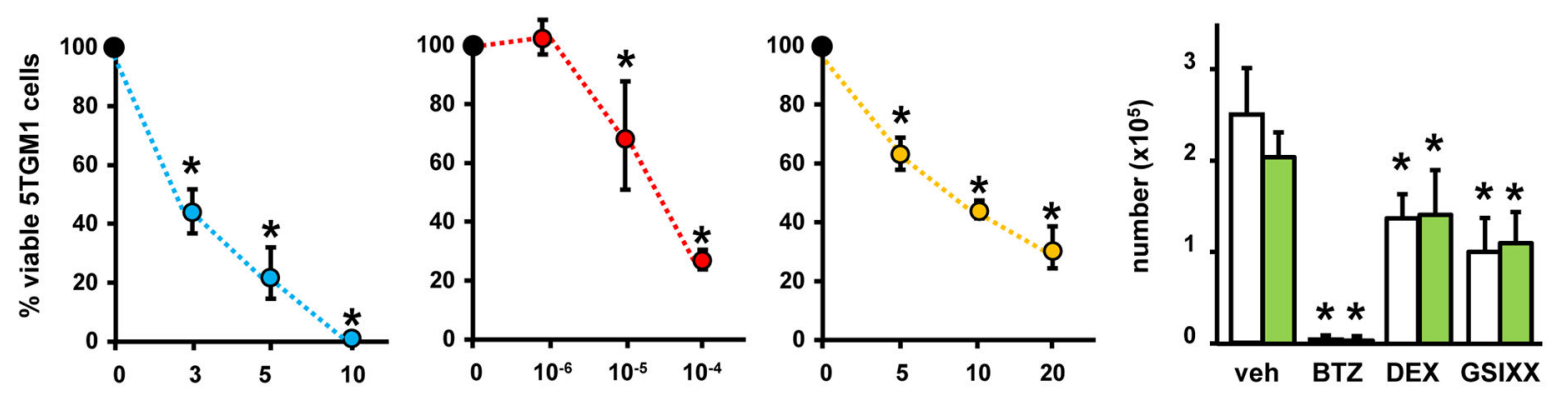

Figure 5. Scl-Ab does not alter the activity of anti-MM drugs

(a) Viability of human JJN3 and murine 5TGM1 myeloma cells treated with Bortezomib (BTZ), Dexamethasone (DEX) or the Notch inhibitor GSIXX alone, or in combination with Scl-Ab (b and c) for 48h; $(n=4)$; *p $\leq 0.05$ vs. vehicle-treated cells (a) or vs. IgG-treated cells (b and $\mathbf{c}$ ); bars represent mean \pm SD. Similar results were observed in a separate experiment. 
physiological levels of Sost/Scl

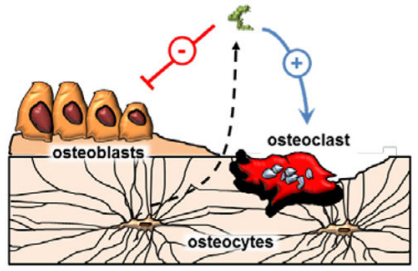

normal bone overexpression of Sost/Scl

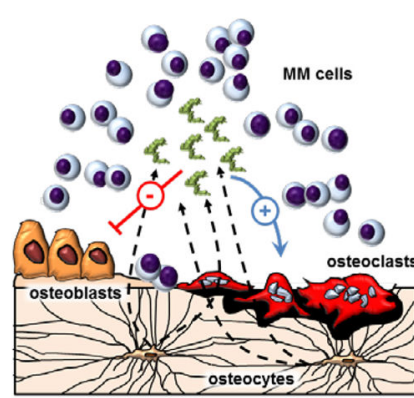

bone colonized by MM cells inhibition of Sost/Scl

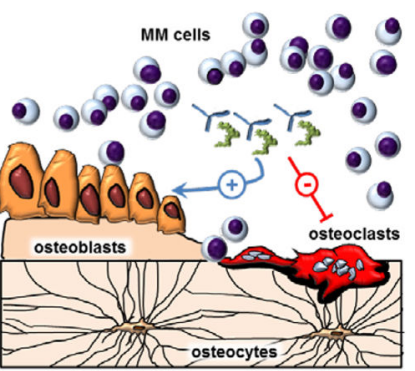

bone colonized by MM cells

\&. Sclerostin $\gamma$ Scl-Ab

Figure 6. Model showing the effects of Scl and Scl-Ab therapy in bone colonized by myeloma cells

In healthy bone, $\mathrm{Scl}$ modulates bone mass by antagonizing Wnt signaling to inhibit bone formation and stimulate bone resorption (left paneI). In MM-colonized bone overproduction of Scl contributes to bone loss and osteolysis by suppressing osteoblasts function and increasing osteoclast numbers (middle panel). Blockade of Scl using Scl-Ab increases osteoblast number and stimulates new bone formation, and results in modest decreases in bone resorption, thus improving MM-induced bone disease (right panel). 